\title{
ON A GENERALIZATION OF CLOSE-TO-CONVEXITY OF COMPLEX ORDER
}

\author{
KHALIDA INAYAT NOOR
}

Abstract. The class $V_{k}$ of bounded boundary rotation is used to generalize the concept of close-to-convexity of complex order. A function $f: f(z)=z+\sum_{n=2}^{\infty} a_{n} z^{n}$, analytic in the unit $\operatorname{disc} E$, belongs to $T_{k}(b), b \neq 0$ (complex) if and only if there exists a function $g \in V_{k}$ such that

$$
\operatorname{Re}\left\{1+\frac{1}{b}\left(\frac{f^{\prime}(z)}{g^{\prime}(z)}-1\right)\right\}>0, \quad z \in E
$$

Some basic properties, rate growth of Hankel determinant and radii problems for the functions in $T_{k}(b)$ are studied.

\section{Introduction}

Let $A$ denote the class of functions $f$ given by

$$
f(z)=z+\sum_{n=2}^{\infty} a_{n} z^{n}
$$

which are analytic in the unit disc $E=\{z:|z|<1\}$. By $S, K, S^{*}$ and $C$, we denote the subclasses of $A$ which are respectively univalent, close-to-convex, starlike and convex in $E$. Let $P$ be the class of analytic functions $h$ given by

$$
h(z)=1+\sum_{n=1}^{\infty} c_{n} z^{n}
$$

with $\operatorname{Re} h(z)>0$ for $z \in E$.

Let $V_{k}, k \geq 2$ be the class of functions of bounded boundary rotation and let $P_{k}$ be the class of functions $p$ analytic in $E$ and have the representation

$$
p(z)=\frac{1}{2} \int_{-\pi}^{\pi} \frac{1+z e^{-i t}}{1-z e^{-i t}} d \mu(t)
$$

Received September 8, 1994.

1991 Mathematics Subject Classification. Primary 30C45.

Key words and phrases. Bounded boundary rotation, starlike, close-to-convex of complex order, univalent, Hankel determinant, radius of convexity. 
where $\mu(t)$ is a function with bounded variation on $[-\pi, \pi]$ which satisfies the conditions

$$
\int_{-\pi}^{\pi} d \mu(t)=2, \int_{-\pi}^{\pi}|d \mu(t)| \leq k .
$$

We note that $P_{2}=P$.

It is known [13] that $P_{k}$ is a convex set. Also $f$, given by (1.1), belongs to $V_{k}$ if and only if $\frac{\left(z f^{\prime}(z)\right)^{\prime}}{f^{\prime}(z)} \in P_{k}$. It is clear that $p \in P_{k}$ if and only if

$$
p(z)=\left(\frac{k}{4}+\frac{1}{2}\right) p_{1}(z)-\left(\frac{k}{4}-\frac{1}{2}\right) p_{2}(z)
$$

where $p_{1}, p_{2} \in P$.

We define the class $P(b)$ as follows.

Difinition $1: 1$. Let $b \neq 0$ be a complex number. Then an analytic function $h$, given by (1.2), is said to belong to $P(b)$, if and only if, there exists a function $p \in P$ such that

$$
h(z)=b p(z)+(1-b) .
$$

We note that, for $0<b<1$,

$$
p(b) \subset P
$$

and $P(1)=P$.

In the following we define a generalized concept of close-to-convexity of complex order.

Definition 1.2. Let $f$ be analytic in $E$ and be given by (1.1). Then $f \in T_{k}(b), k \geq 2$, $b \neq 0$ (complex), if and only if, there exists a function $g \in V_{k}$ such that $\frac{f^{\prime}(z)}{g^{\prime}(z)} \in P(b)$ for $z \in E$.

We note that $T_{k}(1)=T_{k}$, a class of analytic functions introduced and studited in [9] and $T_{2}(1)$ is the class $K$ of close-to-convex functions. Also $T_{2}(b)=K(b)$ consists entirely of close-to-convex functions of complex order introduced in [1] by Al-Amiri and Fernando.

\section{Some Basic Properties of $T_{k}(b)$}

Theorem 2.1. $f \in T_{k}(b)$ if and only if

$$
f^{\prime}(z)=\frac{\left(K_{1}^{\prime}(z)\right)^{\frac{k}{4}+\frac{1}{2}}}{\left(K_{2}^{\prime}(z)\right)^{\frac{k}{4}-\frac{1}{2}}}
$$

where $K_{1}$ and $K_{2}$ are close-to-convex functions of complex order b. 
Proof. From definition 1.2, we have

$$
\begin{aligned}
f^{\prime}(z) & =g^{\prime}(z) h(z), g \in V_{k}, h \in P(b) \\
& =\frac{\left(s_{1}(z) / z\right)^{\frac{k}{4}+\frac{1}{2}}}{\left(s_{2}(z) / z\right)^{\frac{k}{4}-\frac{1}{2}}} h(z), s_{1}, s_{2} \in S^{*}, \text { see }[3] . \\
& =\frac{\left(K_{1}(z)\right)^{\frac{k}{4}+\frac{1}{2}}}{\left(K_{2}(z)\right)^{\frac{k}{4}-\frac{1}{2}}}, \quad K_{1}, K_{2} \in K(b) .
\end{aligned}
$$

Theorem.2.2 Let $0<b_{1}<b_{2}$. Then $T_{k}\left(b_{1}\right) \subset T_{k}\left(b_{2}\right)$.

Proof. Let $f \in T_{k}\left(b_{1}\right)$. Then there exists a function $g \in V_{k}$ such that

$$
\frac{f^{\prime}(z)}{g^{\prime}(z)}=b_{1} h(z)+\left(1-b_{1}\right), h \in P .
$$

Now

$$
1+\frac{1}{b_{2}}\left[\frac{f^{\prime}(z)}{g^{\prime}(z)}-1\right]=\frac{b_{1}}{b_{2}} h(z)+\left(1-\frac{b_{1}}{b_{2}}\right) .
$$

Since $0<b_{1}<b_{2}$, we have $0<\frac{b_{1}}{b_{2}}<1$ and this means $0<\left(1-\frac{b_{1}}{b_{2}}\right)=\alpha_{1}<1$. Hence $R e\left[1+\frac{1}{b_{2}}\left\{\frac{f^{\prime}(z)}{g^{\prime}(z)}-1\right\}\right]>\alpha_{1}>0$. This implies that $f \in T_{k}\left(b_{2}\right)$, and this completes the proof.

We now discuss a geometrical property for the class $T_{k}(b)$. Here we investigate the behaviour of the inclination of the tangent at a point $\omega(\theta)=f\left(r e^{i \theta}\right)$ to the image $\Gamma_{r}$ of the circle $C_{r}=\{z:|z|=r\}, 0 \leq r<1$, and $\theta$ is any number of interval $(0,2 \pi)$ under the mapping by means of a function $f$ from the class $T_{k}(b)$.

We have

$$
\phi(\theta)=\frac{\pi}{2}+\theta+\arg f^{\prime}\left(r e^{i \theta}\right)=\arg \frac{\partial}{\partial \theta} f\left(r e^{i \theta}\right),
$$

and for $\theta_{2}>\theta_{1}, \theta_{1}, \theta_{2} \in[0,2 \pi]$,

$$
\phi\left(\theta_{2}\right)-\phi\left(\theta_{1}\right)=\theta_{2}+\arg f^{\prime}\left(r e^{i \theta_{2}}\right)-\theta_{1}-\arg f^{\prime}\left(r e^{i \theta_{1}}\right) .
$$

Now, since

$$
\theta+\arg f^{\prime}\left(r e^{i \theta}\right)=\theta+\operatorname{Re}\left\{-i \ln f^{\prime}\left(r e^{i \theta}\right)\right\}
$$

then

$$
\frac{\partial}{\partial \theta}\left(\theta+\arg f^{\prime}\left(r e^{i \theta}\right)\right)=\operatorname{Re}\left\{1+\frac{r e^{i \theta} f^{\prime \prime}\left(r e^{i \theta}\right)}{f^{\prime}\left(r e^{i \theta}\right)}\right\}
$$

Therefore

$$
\int_{\theta_{1}}^{\theta_{2}} \frac{\partial}{\partial \theta}\left(\theta+\arg f^{\prime}\left(r e^{i \theta}\right)\right)=\int_{\theta_{1}}^{\theta_{2}} \operatorname{Re}\left\{1+\frac{r e^{i \theta} f^{\prime \prime}\left(r e^{i \theta}\right)}{f^{\prime}\left(r e^{i \theta}\right)}\right\} d \theta .
$$


On the other hand

$$
\begin{aligned}
\int_{\theta_{1}}^{\theta_{2}} \frac{\partial}{\partial \theta}\left\{\theta+\arg f^{\prime}\left(r e^{i \theta}\right)\right\} d \theta & =\theta_{2}+\arg f^{\prime}\left(r e^{i \theta_{2}}\right)-\theta_{1}-\arg f^{\prime}\left(r e^{i \theta_{1}}\right) \\
& =\phi\left(\theta_{2}\right)-\phi\left(\theta_{1}\right) .
\end{aligned}
$$

So, the integral on the left hand side of the last equality characterizes the increment of the angle of the inclination of the tangent to the curve $\Gamma_{r}$ between the points $\omega\left(\theta_{2}\right)$ and $\omega\left(\theta_{1}\right)$ for $\theta_{2}>\theta_{1}$.

We now have the following.

Theorem 2.3. If $f \in T_{k}(b)$ and $0 \leq r<1,|2 b-1|<1$, then for $\theta_{2}>\theta_{1}$, $\theta_{1}, \theta_{2} \in[0,2 \pi]$,

$$
\int_{\theta_{1}}^{\theta_{2}} \operatorname{Re}\left\{1+\frac{r e^{i \theta} f^{\prime \prime}\left(r e^{i \theta}\right)}{f^{\prime}\left(r e^{i \theta}\right)}\right\} d \theta>-\frac{k}{2} \pi+2 \cos ^{-1} \frac{2|b| r}{1-|2 b-1| r^{2}} .
$$

Proof. From definition 1.2, we can write

$$
\operatorname{Re} \frac{\left(z f^{\prime}(z)\right)^{\prime}}{f^{\prime}(z)}=R e \frac{\left(z g^{\prime}(z)\right)^{\prime}}{g^{\prime}(z)}+R e \frac{z h^{\prime}(z)}{h(z)}, g \in V_{k}, h \in P(b) .
$$

With $z=r e^{i \theta}, 0 \leq r<1, \theta \in[0,2 \pi], \theta_{1}<\theta_{2}$, we have

$$
\int_{\theta_{1}}^{\theta_{2}} \operatorname{Re}\left\{1+\frac{r e^{i \theta} f^{\prime \prime}\left(r e^{i \theta}\right)}{f^{\prime}\left(r e^{i \theta}\right)}\right\} d \theta=\int_{\theta_{1}}^{\theta_{2}} \operatorname{Re}\left[1+\frac{r e^{i \theta} g^{\prime \prime}\left(r e^{i \theta}\right)}{g^{\prime}\left(r e^{i \theta}\right)}\right\} d \theta+\int_{\theta_{1}}^{\theta_{2}} \operatorname{Re} \frac{r e^{i \theta} h^{\prime}\left(r e^{r e^{i \theta}}\right)}{h\left(r e^{i \theta}\right)} d \theta .
$$

It is known [3] that, for $g \in V_{k}$,

$$
\int_{\theta_{1}}^{\theta_{2}} \operatorname{Re}\left\{1+\frac{r e^{i \theta} g^{\prime \prime}\left(r e^{i \theta}\right)}{g^{\prime}\left(r e^{i \theta}\right)}\right\} d \theta>-\left(\frac{k}{2}-1\right) \pi
$$

Now in the second integral we observe that

$$
\begin{aligned}
\frac{\partial}{\partial \theta} \arg h\left(r e^{i \theta}\right) & =\frac{\partial}{\partial \theta} \operatorname{Re}\left\{-i \ln h\left(r e^{i \theta}\right)\right\} \\
& =\operatorname{Re}\left\{\frac{r e^{i \theta} h^{\prime}\left(r e^{i \theta}\right)}{h\left(r e^{i \theta}\right)}\right\} .
\end{aligned}
$$

Consequently

$$
\int_{\theta_{1}}^{\theta_{2}} \operatorname{Re}\left\{\frac{r e^{i \theta} h^{\prime}\left(r e^{i \theta}\right)}{h\left(r e^{i \theta}\right)}\right\} d \theta=\arg h\left(r e^{i \theta_{2}}\right)-\arg h\left(r e^{i \theta_{1}}\right)
$$

Hence

$$
\max _{h \in P(b)}\left|\int_{\theta_{1}}^{\theta_{2}} R e \frac{r e^{i \theta} h^{\prime}\left(r e^{i \theta}\right)}{h\left(r e^{i \theta}\right)} d \theta\right|=\max _{h \in P(b)}\left|\arg h\left(r e^{i \theta_{2}}\right)-\arg h\left(r e^{i \theta_{1}}\right)\right| .
$$


Also, from (1.4), we have

$$
1+\frac{1}{b}(h(z)-1)=p(z), \quad p \in P,
$$

and, for $|z|=r<1$, it is well-known that

$$
\left|p(z)-\frac{1+r^{2}}{1-r^{2}}\right| \leq \frac{2 r}{1-r^{2}}
$$

From this, we have

$$
\left|h(z)-\frac{1+(2 b-1) r^{2}}{1-r^{2}}\right| \leq \frac{2|b| r}{1-r^{2}}
$$

Thus the values of $h(z)$ are contained in the circle of Apollonius whose diameter is the line segment from $\frac{1+(2 b-1) r}{1+r}$ to $\frac{1-(2 b-1) r}{1-r}$. The circle is centered at the point $\frac{1+(2 b-1) r^{2}}{1+r^{2}}$ and has the radius $\frac{1|b| r}{1-r^{2}}$. So $|\arg h(z)|$ attains its maximum at points where a ray from the origin is tangent to the circle, that is, when

$$
\arg h(z)= \pm \sin ^{-1} \frac{2|b| r}{1-|(2 b-1)| r^{2}}
$$

From the above observations, we see that

$$
\begin{aligned}
\max _{h \in P(b)}\left|\int_{\theta_{1}}^{\theta_{2}} R e \frac{r e^{i \theta} h^{\prime}\left(r e^{i \theta}\right)}{h\left(r e^{i \theta}\right)} d \theta\right| & \leq 2 \sin ^{-1} \frac{2|b| r}{1-|2 b-1| r^{2}} \\
& =\pi-2 \cos ^{-1} \frac{2|b| r}{1-|2 b-1| r^{2}} .
\end{aligned}
$$

Using (2.2) and (2.4) in (2.1), we obtain the required result.

Remark 2.1. If $f \in T_{k}(b)$ and $b$ is real, then it can easily be shown that, for $\theta_{2}>\theta_{1}, z=r e^{i \theta}$

$$
\int_{\theta_{1}}^{\theta_{2}} \operatorname{Re}\left\{1+\frac{r e^{i \theta} f^{\prime \prime}\left(r e^{i \theta}\right)}{f^{\prime}\left(r e^{i \theta}\right)}\right\} d \theta>-\left(\frac{k}{2}-1+|b|\right) .
$$

For $\beta_{1} \geq 0$, the class $K\left(\beta_{1}\right)$ has been introduced in [5]. We notice that, if $b$ is real,

(i) $T_{k}(b) \subset K\left(\frac{k}{2}+|b|-1\right)$

(ii) $T_{k}(b)$ consists of univalent functions for $k+2|b| \leq 4$ whilst $f \in T_{k}(b)$ for $k+2|b|>4$ need not be finitely-valent.

(iii) It can easily be seen that $T_{k}(b)$ forms a subset of a linear-invariant family of order $\left(\frac{k}{2}+|b|\right)$.

From (2.3) and the well-known result

$$
\left|\arg g^{\prime}(z)\right| \leq k \sin ^{-1} r
$$


for $g \in V_{k}$, we have the following.

Theorem 2.4. Let $f \in T_{k}(b),|2 b-1| \leq 1$. Then

$$
\left|\arg f^{\prime}(z)\right| \leq k \sin ^{-1} r+\sin ^{-1} \frac{2|b| r}{1-|2 b-1| r^{2}} .
$$

Next we prove a distortion theorem for $T_{k}(b)$.

Theorem 2.5. Let $f \in T_{k}(b)$. Then

$$
\frac{(1-|2 b-1| r)(1-r)^{\frac{k}{2}-1}}{(1+r)^{\frac{k}{2}+2}} \leq\left|f^{\prime}(z)\right| \leq \frac{(1+r)^{\frac{k}{2}-1}(1+|2 b-1| r)}{(1-r)^{\frac{k}{2}+2}}
$$

The equality is attained for the function $f_{0} \in T_{k}(b)$ defined by

$$
f_{0}^{\prime}(z)=\frac{\left(1+\delta_{1} z\right)^{\frac{k}{2}-1}}{\left(1-\delta_{2} z\right)^{\frac{k}{2}+2}}\left(1+(2 b-1) \delta_{1} z\right), \quad\left|\delta_{1}\right|=\left|\delta_{2}\right|=1 .
$$

The proof is immediate when we use the distortion theorems for $g \in V_{k}$, see [13] and for $h \in P(b)$,

$$
\frac{1-|2 b-1| r}{1+r} \leq|h(z)| \leq \frac{1+|2 b-1| r}{1-r} .
$$

\section{Speical Cases.}

(i) For $k=2, f$ is close-to-convex of complex order $b$ and we have

This reult is proved in [1].

$$
\frac{1-|2 b-1| r}{(1+r)^{3}} \leq\left|f^{\prime}(z)\right| \leq \frac{1+|2 b-1| r}{(1-r)^{3}}
$$

(ii) For $b=1$, we obtain the sharp bounds for $f \in T_{k}$ established in [9].

Theorem 2.6.(Covering theorem). The image of $E$ under functions in $T_{k}(b)$ contains the schlicht disc

$$
|z|<\frac{k+1-|2 b-1|}{k(k+2)} \text {. }
$$

Proof. Let $d_{r}$ denote the radius of the largest schlicht disc centered at the origin contained in the image of $|z|<r$ under $f(z)$. Then there is a point $z_{0},\left|z_{0}\right|=r$ such that $\left|f\left(z_{0}\right)\right|=d_{r}$. The ray from 0 to $f\left(z_{0}\right)$ lies entirely in the image of $E$ and the inverse image of this way is a curve in $|z|<r$.

Thus

$$
d_{r}=\left|f\left(z_{0}\right)\right|=\int_{c}\left|f^{\prime}(z)\right||d z| \geq \int_{0}^{r} \frac{\left(1-b_{1} t\right)}{1+t}\left(\frac{1-t}{1+t}\right)^{\frac{k}{2}-1} \cdot \frac{d t}{(1+t)^{2}}
$$


where $b_{1}=|2 b-1|$.

Let $\frac{1-t}{1+t}=\xi$. Then $\frac{-2}{(1+t)^{2}} d t=d \xi$.

So

$$
\begin{aligned}
\left|f\left(z_{0}\right)\right| & \geq-\frac{1}{4} \int_{0}^{\frac{1-r}{1+r}}\left(1-b_{1}\right) \xi^{\frac{k}{2}-1} d \xi-\frac{1}{4} \int_{0}^{\frac{1-r}{1+r}}\left(1+b_{1}\right) \xi^{\frac{k}{2}} d \xi \\
& =-\frac{1}{2}\left(\frac{1-r}{1+r}\right)^{\frac{k}{2}}\left[\frac{\left(1+b_{1}\right)}{k+2}\left(\frac{1-r}{1+r}\right)+\frac{\left(1-b_{1}\right)}{k}\right]+\left(\frac{k+\left(1-b_{1}\right)}{k(k+2)}\right) .
\end{aligned}
$$

Now, by letting $r \rightarrow 1$, we obtain the required result.

\section{Hankel Determinant Problem for $T_{k}(b)$}

Hankel determinant of $f \in A$ and given by (1.1) is defined, for $q \geq 1, n \geq 1$, by

$$
h_{q}(n)=\left|\begin{array}{lll}
a_{n} & a_{n+1} \ldots & a_{n+q-1} \\
a_{n+1} & \ldots & \ldots \\
\vdots & & \\
a_{n+q-1} & \cdots & a_{n+2 q-2}
\end{array}\right| .
$$

For $f \in S^{*}$, Pommerenke [16] solved this problem completely. He showed that, if $f \in S^{*}$, $H_{q}(n)=0(1) n^{2-q}$ and the exponent $(2-q)$ is best possible.

We shall investigate the rate of growth of $H_{q}(n)$ for $f \in T_{k}(b)$. We first prove the following.

Theorem 3.1. Let $f \in T_{k}(b), k>3$ and be given by (1.1). Then, for $m=0,1,2, \ldots$, there are numbers $\gamma_{m}$ and $c_{m \mu}(\mu=0, \ldots, m)$ that satisfy $\left|c_{m o}\right|=\left|c_{m m}\right|=1$, and

$$
\sum_{k=0}^{\infty} \gamma_{k} \leq 3, \quad 0 \leq \gamma_{m} \leq \frac{2}{m+1}
$$

such that

$$
\sum_{m u=0}^{\infty} c_{m \mu} a_{n+\mu}=O(1) \cdot n^{\gamma_{m}+\frac{k}{2}-2}, \quad(n \rightarrow \infty) .
$$

The bounds (3.1) are best possible.

Proof. Since $f \in T_{k}(b)$, there exists $g \in V_{k}$ such that, for $z \in E$ and $h \in P(b)$, we have

$$
f^{\prime}(z)=g^{\prime}(z) h(z)
$$

Let $\tilde{K}(\beta)$ be the class of strongly close-to-convex functions of order $\beta$ in the sense of Pommerenke [14]. It is known [4] that, for all $k>2, V_{k}$ is properly contained in $\tilde{K}(\beta)$ and $\beta=\left(\frac{k}{2}-1\right)$. From this it follows that, if $g \in V_{k}, k>2$, then

$$
z g^{\prime}(z)=s(z) p^{\frac{k}{2}-1}(z)
$$


for some $s \in S^{*}, p \in P$.

Thus we can write $(3.2)$ as

$$
z f^{\prime}(z)=s(z) p^{\frac{k}{2}-1}(z) h(z)
$$

Now $s$ can be represented as

$$
s(z)=z \exp \left[\int_{0}^{2 \pi} \log \frac{1}{1-z e^{-i t}} d \mu(t)\right]
$$

where $\mu(t)$ is an increasing function and $\mu(2 \pi)-\mu(0)=2$.

Let $\alpha_{1} \geq \alpha_{2} \geq \cdots$ be the jumps of $\mu(t)$ and $t=\theta_{1}, \theta_{2}, \ldots$ be the values at which these jumps occur. We may assume that $\theta_{1}=0$. Then $\alpha_{1}+\alpha_{2}+\cdots \leq 2$ and $\alpha_{1}+\alpha_{2}+\cdots+\alpha_{q}=2$ for some $q$ if and only if $s$ is of the form

$$
s(z)=z \prod_{j=1}^{q}\left(1-e^{-i \theta_{j}} z\right)^{-2 / q} .
$$

We define $\phi_{m}$ by

$$
\phi_{m}(z)=\prod_{\mu=1}^{m}\left(1-e^{i \theta \mu} z\right) .
$$

We consider three cases as in [16] and define $\eta_{m}$, for each case respectively, as follows.

(i) $0 \leq \alpha_{1} \leq 1$ and $\eta_{m}=\alpha_{m+1}(m=0,1,2, \ldots)$

(ii) $1<\alpha_{1}<\frac{3}{2}$ and $\eta_{0}=\alpha_{1}, \eta_{1}=\max \left(\alpha_{1}-1, \alpha_{2}\right), \eta_{2}=\max \left(\alpha_{1}-1, \alpha_{3}\right), \eta_{m}=\alpha_{m}$ for $m \geq 3$.

(iii) $\frac{3}{2} \leq \alpha_{1} \leq 2$ and $\eta_{0}=\alpha_{1}, \eta_{1}=\max \left(\alpha_{1}-1, \alpha_{2}\right), \eta_{m}=\alpha_{m}(m \geq 2)$.

Then the first part, that is the the bounds (3.1), follows similarly as in [16]. For the rest, we need the following.

Lemma 3.1. [16] Let $\theta_{1}<\theta_{2}<\cdots<\theta_{q}<\theta_{1}+2 \pi$ and let $\lambda_{1}, \ldots, \lambda_{q}$ be real, $\lambda>0$, $\lambda \geq \lambda_{j}(j=1, \ldots, q)$. If

$$
\psi(z)=\prod_{j=1}^{q}\left(1-e^{-i \dot{\theta}_{j}} z\right)^{-\lambda_{j}}=\sum_{n=1}^{\infty} b_{n} z^{n},
$$

then

$$
b_{n}=O(1) \cdot n^{\lambda-1} \quad \text { as } n \rightarrow \infty .
$$

We now complete the proof of theorem 3.1. We write

$$
\phi_{m}(z)=\sum_{\mu=0}^{m} c_{m \mu} z^{m-\mu}
$$


and

$$
\phi_{m}(z) \cdot z f^{\prime}(z)=\sum_{n=1}^{m} b_{m n} z^{n+m}+\sum_{n=1}^{\infty}(n+m) a_{m n} z^{z+m}
$$

where

$$
\begin{aligned}
b_{m n} & =\sum_{\nu=0}^{n}(n+\nu) c_{m-\nu} a_{n-\nu} \\
a_{m n} & =\sum_{m \mu} c_{m \mu} a_{n+\mu},\left|c_{m_{0}}\right|=\left|c_{m m}\right|=1
\end{aligned}
$$

First let $s$ in (3.3) be not of the form (3.4). Then $\alpha_{1}+\alpha_{2}+\cdots+\alpha_{k}<2$ for $k \geq 1$ and in particular $\alpha_{1}<2$. Hence the number $\eta_{m}$ defined as before satisfy

$$
\eta_{m}<\frac{2}{m+1}, \quad \eta_{0}+\eta_{1}+\cdots<3
$$

For $m \geq 0$, let

$$
\delta_{m}=\frac{1}{3} \min \left\{\frac{2}{m+1}-\eta_{m}, \frac{1}{2^{m+1}}\left(3-\sum_{k=0}^{m} \eta_{k}\right)\right\}
$$

and

$$
\gamma_{m}=\eta_{m}+2 \delta_{m}
$$

Then $\delta_{m}>0$ and $\gamma_{m}<\frac{2}{m+1}, \gamma_{0}+\gamma_{1}+\cdots<3$. Now, it can easily be shown [15] that in each case $(i),(i i),(i i i)$,

$$
\max _{|z|=r}\left|\phi_{m}(z) g(z)\right|=O(1) \cdot(1-r)^{-\eta_{m}-\delta_{m}}
$$

Thus, from (3.6), (3.3) and Cauchy integral fromula, we have

$$
\begin{aligned}
(n+m)\left|a_{m n}\right| & \leq \frac{1}{r^{n+m}}\left(\frac{1}{2 \pi} \int_{0}^{2 \pi}\left|\phi_{m}(z) s(z) p^{\frac{k}{2}-1}(z) h(z)\right| d \theta\right) \\
& \leq \frac{1}{2 \pi r^{n+m}} \max \left|\phi_{m}(z) s(z)\right|\left(\int_{0}^{2 \pi}\left|p^{\frac{k}{2}-1} h(z)\right| d \theta\right) \\
& \leq \frac{1}{r^{n+m}} \cdot \max \left|\phi_{m}(z) s(z)\right|\left(\frac{1}{2 \pi} \int_{0}^{2 \pi}\left|p^{k-2}(z)\right| d \theta\right)^{\frac{1}{2}}\left(\frac{1}{2 \pi} \int_{0}^{2 \pi}|h(z)|^{2} d \theta\right)^{\frac{1}{2}} .
\end{aligned}
$$

We shall need the following two lemmas.

Lemma 3.2. [6] Let $p \in P$ for $z \in E$. Then, for $\lambda>1$,

$$
\int_{0}^{2 \pi}\left|p\left(r e^{i \theta}\right)\right|^{\lambda} d \theta<c(\lambda) \frac{1}{(1-r)^{\lambda-1}} .
$$


Lemma 3.3. [12] Let $h \in P(b)$ in $E$ and be given by (1.2). Then

$$
\frac{1}{2 \pi} \int_{0}^{2 \pi} \mid h\left(\left.r e^{i \theta}\right|^{2} d \theta \leq \frac{1+\left(4|b|^{2}-1\right) r^{2}}{1-r^{2}}\right.
$$

Using these lemmas in (3.8), we have for $k>3$

$$
(n+m)\left|a_{m n}\right|=O(1) \cdot(1-r)^{-\eta_{m}-\delta_{m}-\left(\frac{k}{2}-1\right)} \quad(r \rightarrow 1),
$$

which implies

$$
a_{m n}=O(1) n^{\gamma_{m}+\frac{k}{2}-2} \quad(n \rightarrow \infty) .
$$

We now consider the case when $s$ in (3.3) has the form (3.4), that is, $\alpha_{1}+\alpha_{2}+\cdots+\alpha_{q}=2$ with $\gamma_{m}=\eta_{m}$. It follows that

$$
\gamma_{m} \leq \frac{2}{m+1}, \gamma_{0}+\gamma_{1}+\cdots \leq 3
$$

and

$$
\gamma_{m}=\frac{2}{m+1} \text { implies that } m=q-1, \alpha_{1}=\cdots=\alpha_{q} .
$$

Thus, for $k>3$

$$
\begin{aligned}
\left(n+n_{\iota}\right)\left|a_{m n}\right| & \leq \frac{1}{2 \pi r^{n+m}} \int_{0}^{2 \pi}\left|\phi_{m}(z) s(z) p^{\frac{k}{2}-1}(z) h(z)\right| d \theta \\
& \leq \frac{1}{r^{n+1}}\left(\frac{1}{2 \pi} \int_{0}^{2 \pi}\left|\phi_{m}(z) s(z) p^{\frac{k}{2}-1}(z)\right|^{2} d \theta\right)^{\frac{1}{2}}\left(\frac{1}{2 \pi} \int_{0}^{2 \pi}|h(z)|^{2} d \theta\right)^{\frac{1}{2}}
\end{aligned}
$$

Now

$$
\frac{1}{2 \pi} \int_{0}^{2 \pi}\left|\phi_{m}(z) s(z)\right|^{2}|p(z)|^{k-2} d \theta \leq\left(\frac{1}{2 \pi} \int_{0}^{2 \pi}\left|\phi_{m}(z) s(z)\right|^{4} d \theta\right)^{\frac{1}{2}}\left(\frac{1}{2 \pi} \int_{0}^{2 \pi}|p(z)|^{2 k-4} d \theta\right)^{\frac{1}{2}}
$$

When we wite $\left|\phi_{m}(z)\right|^{4}$ in the form (3.5), the exponents $\left(-\lambda_{j}\right)$ satisfy $\lambda_{j} \leq 4 \gamma_{m}(j=$ $1, \ldots, q: m>0)$. Hence, by using lemma 2.1 , we have

$$
\int_{0}^{2 \pi}\left|\phi_{m}(z) s(z)\right|^{4} d \theta \leq A_{1} n^{4 \gamma_{m}-1} \quad(n \rightarrow \infty) .
$$

Also, since $k>3$, we use Lemma 3.2 to have

$$
\frac{1}{2 \pi} \int_{0}^{2 \pi}|p(z)|^{2 k-4} d \theta \leq A_{2} n^{2 k-5} \quad(n \rightarrow \infty)
$$

Thus, from (3.10), (3.11) and (3.12), we have

$$
\frac{1}{2 \pi} \int_{0}^{2 \pi}\left|\phi_{m}(z) s(z)\right|^{2}|p(z)|^{k-2} d \theta \leq A n^{2 \gamma_{m}+k-3} \text {. }
$$


Hence, using: (3.13) and Lemma 3.3 in (3.9), we obtain

$$
(n+m)\left|a_{m n}\right| \leq B(k, b) n^{\gamma_{m}+\frac{k}{2}-1} \quad(n \rightarrow \infty),
$$

and this gives us

$$
a_{m n}=O(1) \cdot n^{\gamma_{m}+\frac{k}{2}-2}
$$

The function $s_{0}: s_{0}(z)=z\left(1-z^{q}\right)^{-\frac{2}{q}}=\sum_{\nu=0}^{\infty}\left(\frac{2}{q}+\nu-1\right) z^{\nu q+1}$ shows that the bounds in (3.1) are best possible. This completes the proof of theorem 3.1.

We can now easily prove the following result.

Theorem 3.2. Let. $f \in T_{k}(b), k>3$ and $f$ be given by (1.1). Then, for $q \geq 1, n \geq 1$,

$$
H_{q}(n)=O(1) \cdot n^{2+\left(\frac{k}{2}-2\right) q} .
$$

The exponent $\left[2+\left(\frac{k}{2}-2 q\right]\right.$ is best possible.

In particular, for $q=1, k>3$

$$
H_{1}(n)=a_{n}=O(1) \cdot n^{\frac{k}{2}} \quad(n \rightarrow \infty) .
$$

Remark 3.1. From remark 2.1, it is clear that, for $b$ real, $T_{k}(b) \subset K\left(\frac{k}{2}+|b|-1\right)$. Thus for $f \in T_{k}(b), b$ real, we can write

$$
z f^{\prime}(z)=s(z) p(z)^{\frac{k}{2}+|b|-1}, s \in S^{*}, p \in P .
$$

Following the same techniques of Theorems 3.1 and 3.2 together with the remark 3.1 , we have the following.

Theorem 3.3. Let $f \in T_{k}(b)$, b real,$k+2|b|>3$. Then, for $q \geq 1, n \geq 1$

$$
\left.H_{q}(n)=O(1) \cdot n^{2+q\left(\frac{k}{2}+|b|-3\right.}\right) .
$$

\section{Some Radii Problems}

In the following we find the radius of convexity for $f \in T_{k}(b)$.

Theorem 4.1. Let $f \in T_{k}(b)$. Then $f$ maps $|z|<r_{0}$ onto a convex domain, where $r_{0}$ is the least positive root of the equation

$$
T(r)=(1+\operatorname{Re} \mu)-(1+k)(1+\operatorname{Re} \mu) r-(1+k)(1-\operatorname{Re} \mu) r^{2}+(1-\operatorname{Re} \mu) r^{3}=0
$$

where $\mu=\frac{1-b}{b}$ and Re $\mu \geq 0$. 

by

This results is best possible for $b=1$ where the extremel function $F_{k} \in T_{k}(1)$ is given

$$
F_{k}(z)=\frac{1}{k+2}\left[\left(\frac{1+z}{1-z}\right)^{\frac{k}{2}+1}-1\right] .
$$

Also, for $b=1, k=2, r_{0}$ is the radius of convexity for $f \in K$ and in this case $r_{0}=2-\sqrt{3}$.

Proof. We can write

$$
\begin{aligned}
z f^{\prime}(z) & =z g^{\prime}(z) h(z), g \in V_{k}, h \in P(b) \\
& =z g^{\prime}(z)[b p(z)+(1-b)], p \in P .
\end{aligned}
$$

Differentiating logarithmically, we have

$$
\frac{\left(z f^{\prime}(z)\right)^{\prime}}{f^{\prime}(z)}=\frac{\left(z g^{\prime}(z)\right)^{\prime}}{g^{\prime}(z)}+\frac{z p^{\prime}(z)}{p(z)+\frac{1-b}{b}} .
$$

Thus

$$
\operatorname{Re} \frac{\left(z f^{\prime}(z)\right)^{\prime}}{f^{\prime}(z)} \geq \operatorname{Re} \frac{\left(z g^{\prime}(z)\right)^{\prime}}{g^{\prime}(z)}-\left|\frac{z p^{\prime}(z)}{p(z)+\mu}\right|, \quad \mu=\left(\frac{1-b}{b}\right) .
$$

It is known [13] that for $g \in V_{k}$

$$
R e \frac{\left(z g^{\prime}(z)\right)^{\prime}}{g^{\prime}(z)} \geq \frac{r^{2}-k r+1}{1-r^{2}}, z=r e^{i \theta}, 0 \leq r<1,
$$

and for $p \in P, R e \mu \geq 0$,

$$
\left|\frac{z p^{\prime}(z)}{p(z)+\mu}\right| \leq \frac{2 r}{(1-r)[1+r+\operatorname{Re} \mu(1-r)]}, \operatorname{see}[7] .
$$

Hence we have

$$
\begin{aligned}
\operatorname{Re} \frac{\left(z f^{\prime}(z)\right)^{\prime}}{f^{\prime}(z)} & \geq \frac{r^{2}-k r+1}{1-r^{2}}-\frac{2 r}{(1-r)[1+r+\operatorname{Re} \mu(1-r)]} \\
& =\frac{(1+\operatorname{Re} \mu)-(k+1)(1+\operatorname{Re} \mu) r-(1+k)(1-\operatorname{Re} \mu) r^{2}+(1-\operatorname{Re} \mu) r^{3}}{\left(1-r^{2}\right)[1+\gamma+\operatorname{Re} \mu(1-r)]} .
\end{aligned}
$$

This implies that $\operatorname{Re} \frac{\left(z f^{\prime}(z)\right)^{\prime}}{f^{\prime}(z)}>0$ for $|z|<r_{0}$ where $r_{0}$ is the least positive root of $T(r)=0$ give by (4.1).

We note here that $T(0)=1+\operatorname{Re} \mu>0$ and $T(1)=-2 k<0$ which means that $T(r)=0$ has at least one zero in $(0,1)$.

Theorem 4.2. Let $f \in T_{k}(b)$ and $F$ be defined, for $0<\alpha<1$, by

$$
F(z)=\frac{1}{\alpha} z^{1-\frac{1}{\alpha}} \int_{0}^{z} \xi^{\frac{1}{\alpha}-2} f(\xi) d \xi
$$


Then $F$ is close-to-convex of complex order $b$ for $|z|<r_{1}$, where

$$
r_{1}=\frac{1}{2}\left[k-\sqrt{k^{2}-4}\right] \text {. }
$$

This result is sharp.

Proof. Since $f \in T_{k}(b)$, there exists a function $g \in V_{k}$ such that $\frac{f^{\prime}(z)}{g^{\prime}(z)} \in P(b)$. Let

$$
G(z)=\frac{1}{\alpha} z^{1-\frac{1}{\alpha}} \int_{0}^{z} \xi^{\frac{1}{\alpha}-2} g(\xi) d \xi
$$

Then, from a special case of a result proved in [11], we see that $G$ is convex for $|z|<r_{1}$ and this radius is best possible.

Now

$$
\begin{aligned}
\frac{F^{\prime}(z)}{G^{\prime}(z)} & =\frac{z^{\frac{1}{\alpha}-1} f(z)-\left(\frac{1}{\alpha}-1\right) \int_{0}^{z} \xi^{\frac{1}{\alpha}-2} f(\xi) d \xi}{z^{\frac{1}{\alpha}-1} g(z)-\left(\frac{1}{\alpha}-1\right) \int_{0}^{z} \xi^{\frac{1}{\alpha}-2} g(\xi) d \xi} \\
& =\frac{\int_{0}^{z} \xi^{\frac{1}{\alpha}-1} f^{\prime}(\xi) d \xi}{\int_{0}^{z} \xi^{\frac{1}{\alpha}-1} g^{\prime}(\xi) d \xi}=\frac{N(z)}{D(z)}
\end{aligned}
$$

and

$$
\frac{N^{\prime}(z)}{D^{\prime}(z)}=\frac{f^{\prime}(z)}{g^{\prime}(z)} \in P(b)
$$

Since $g \in V_{k}$, we know that $g$ is convex for $|z|<r_{1}$ and so $z g^{\prime}$ is starlike for $|z|<r_{1}$. Now, using a similar technique of Libera [8], we can easily show that $\frac{N(z)}{D(z)} \in P(b)$ for $|z|<r_{1}$ where $r_{1}$ is given by (4.2). This completes the proof.

We have the following special cases.

(i) For $k=2, f$ is close-to-convex of complex order $b$. Then $F$ is also close-to-convex of complex order $b$ in $E$.

(ii) For $b=1, f \in T_{k}$. Then $F$ is close-to-convex (hence univalent) in $|z|<r_{1}$.

(iii) When $b=1, k=2, \frac{1}{\alpha}$ a positive integer, we obtain a result proved by Bernardi [2].

(iv) Libera [8] proved this result with $b=1, k=2$ and $\alpha=\frac{1}{2}$.

Theorem 4.3. Let $f \in T_{k}(b)$ with respect to $h \in V_{k}$. Let $g \in V_{k}$ and for $\alpha, \beta$ positively real with $\alpha+\beta=1$, let

$$
F(z)=\int_{0}^{z}\left(f^{\prime}(\xi)\right)^{\alpha}\left(g^{\prime}(\xi)\right)^{\beta} d \xi
$$

and

$$
H(z)=\int_{0}^{z}\left(h^{\prime}(\xi)\right)^{\alpha}\left(g^{\prime}(\xi)\right)^{\beta} d \xi
$$


Then $F \in T_{k}$ with respect to $H$ for $|z|<r_{2}$ where $r_{2}$ is given by

$$
r_{2}=\left[\frac{1}{|b|+\sqrt{|b|^{2}-2 R e b+1}}\right] \text {. }
$$

Proof. We first note that $H \in V_{k}$ since

$$
\begin{aligned}
\frac{\left(z H^{\prime}(z)\right)^{\prime}}{H^{\prime}(z)} & =\frac{\alpha\left(z h^{\prime}(z)\right)^{\prime}}{h^{\prime}(z)}+\frac{\beta\left(z g^{\prime}(z)\right)^{\prime}}{g^{\prime}(z)} \\
& =\alpha p_{1}(z)+\beta p_{2}(z), p_{1}, p_{2} \in P_{k} \\
& =p_{3}(z), p_{3} \in P_{k} \text { as } P_{k} \text { is a convex set. }
\end{aligned}
$$

Now

$$
\frac{F^{\prime}(z)}{H^{\prime}(z)}=\frac{\left(f^{\prime}(z)\right)^{\alpha}\left(g^{\prime}(z)\right)^{\beta}}{\left(h^{\prime}(z)\right)^{\alpha}\left(g^{\prime}(z)\right)^{\beta}}=\left(\frac{f^{\prime}(z)}{h^{\prime}(z)}\right)^{\alpha}=(p(z))^{\alpha} .
$$

Since $p \in P$ for $|z|<r_{2}$ where $r_{2}$ is given by (4.3), see [1], it follows that $p^{\alpha} \in P$ for $|z|<r_{2}$ which implies that $F \in T_{k}$ for $|z|<r_{2}$.

Theorem 4.4. Let $f \in V_{k}$ and let

$$
F(z)=b z^{2-\frac{1}{b}}\left[z^{\frac{1}{b}-1} f(z)\right]^{\prime} .
$$

Then $F \in T_{k}(b)$ for all $|z|<r_{1}$ where $r_{1}$ is given by (4.2). This result is sharp.

Proof. Let $F^{\prime}(z)=b\left[\left(\frac{1}{b}-1\right) f^{\prime}(z)+\left(z f^{\prime}(z)\right)^{\prime}\right]$

Then

$$
\frac{F^{\prime}(z)}{f^{\prime}(z)}=b\left[\frac{\left(z f^{\prime}(z)\right)^{\prime}}{f^{\prime}(z)}+\left(\frac{1}{b}-1\right)\right]=b H(z)+(1-b)
$$

Since $H \in P_{k}$, it follows that $H \in P$ for $|z|<r_{1}$ and the radius $r_{1}$ is best possible, see [13]. This implies that, for $|z|<r_{1} \cdot F \in T_{k}(b)$.

Remark 4.1. Since $r_{1}$ is the radius of convexity for $g \in V_{k}$, we can conclude that $f \in T_{k}(b)$ is close-to-convex of complex order $b$ for $|z|<r_{1}$ where $r_{1}$ is given by (4.2).

Following essentially the same technique used in [10], we can prove:

Theorem 4.5. Let $F \in T_{2}(b)$ and let, for $0<\lambda<1$,

$$
f(z)=(1-\lambda) F(z)+\lambda z F^{\prime}(z) .
$$

Then $f \in T_{2}(b)$ for $|z|<r_{\lambda}$, where

$$
r_{\lambda}=\left[\frac{1}{2 \lambda+\sqrt{4 \lambda^{2}-2 \lambda+1}}\right] .
$$

This result is best possible. 


\section{References}

[1] H. S. Al-Amiri and T. S. Fernando, "On close-to-convex functions of complev order," Int. J. Math. and Math. Sci., 13(1990), 321-330.

[2] S. D. Bernardi, "Convex and starlike univalent functions," Trans. Amer. Math. Soc., 135(1969), 429-446.

[3] D. A. Brannan, "On functions of bounded boundary rotation," Proc. Edin. Math. Soc., 2(1968/69), 339-347.

[4] D. A. Brannan, J. G. Clunie and W. E. Kirwan, "On the coefficient problem for functions of bounded boundary rotation," Ann. Acad. Sci. Fenn. Series AI, 523(1973), 1-18.

[5] A. W. Goodman, "On close-to-convex functions of higher order," Ann. Univ. Sci. Budapest Eötvös Sect. Maith., 15(1972), 17-30.

[6] W. K. Hayman, "On functions with positive real part," J. Lond. Math. Soc., 36(1961), $34-48$.

[7] R. J. Libera, "Some radius of convexity problems," Duke Math. J., 31(1964), 143-158.

[8] R. J. Libera, "Some classes of regular univalent functions," Proc. Amer. Math. Soc., 16(1965), .753-758.

[9] K. I. Noor, "On a generalization of close-to-convexity," Int. J. Math. and Math. Sci., 6(1983), 327-334.

[10] K. I. Noor, F. Al-Oboudi and N. Al-Dihan, "On the radius of univalence of convex combinations of analytic functions," Int. J. Math. and Math. Sci., 6(1983), 335-340.

[11] K. I. Noor, "On some analytic functions of class $P_{k}(\alpha)$, " C. R. Math. Rep. Acad. Sci., Canada, 12(1990), 69-74.

[12] K. I. Noor, "On close-to-convex functions of complex order and related subclasses, Analysis," Geometry and Groups: A Riemann Legacy Volume (ed. H. M. Srivastava and Th. M. Rassias) Hadronic Press, U. S. A., (1994), 313-335.

[13] B. Pinchuk, "Functions with bounded boundary rotation," I. J. Math., 10(1971), 7-16.

[14] Ch. Pommerenke, "On close-to-convex analytic functions," Trans. Amer. Math. Soc., 114(1965), 176-186.

[15] Ch. Pommerenke, "On starlike and close-to-convex functions," Proc. Amer. Math. Soc., 13(1963), 290-304.

[16] Ch. Pommerenke, "On the coefficients and Hankel determinants of univalent functions," J. Lond. Math. Soc., 41(1966), 111-122.

Mathematics Department, College of Science, P. O. Box 2455, King Saud University, Riyadh 11451, Saudi Arabia. 